\title{
Pseudomonas aeruginosa and it's One of the Virulence Factors: Rhamnolipid
}

\author{
Bukavaz S* \\ Department of Environmental Health, Trakya University, Turkey
}

*Corresponding author: Asisstant Professor Sebnem Bukavaz, Department of Environmental Health in Health Services Vocational College, Trakya University Balkan Campus Edirne TR22030, Turkey, Email: sbukavaz@trakya.edu.tr

\section{Editorial}

Volume 4 Issue 1

Received Date: January 18, 2021

Published Date: February 02, 2021

DOI: $10.23880 /$ oajmms-16000133

\section{Editorial}

Oppurtunistic pathogen Pseudomonas aeruginosa is known as most adaptive bacteria to diversity of ecological niches thanks to their multifactorial regulating system and non- stringent metabolic requirements. Adaptation and coordination of gene expression is particularly crucial in pathogenesis that allowed them to colonize on various host environment conditions are recognized and responded by two component system and Quorum sensing [1]. This oppurtunistic pathogen nearly never infcets healthy individuals, but particularly in patients with severe burns and in cancer, AIDS patients who are immunosuppressed. The case fatality rate in these patients is near $50 \%$ and $P$. aeruginosa is famous as a major cause of nosocomial infections associated with invasive devices, mechanical ventilation, burn, wounds or surgery in immunocompetent host [2]. Currently it causes Carbapenem resistance with the rest very few treatment options [3]. Since then, the genome sequence of the widely studied $P$. aeruginosa strain PA01 revealed that is possesses a large number of genes that are involved in regulation, catabolism, transport and efflux of organic compounds, as well as several putative chemotaxis systems, all of which potentially contribute to the remarkable ability of this bacterium to adapt to a wide range environmental niches. The PAO1 strain has been and is still the major reference for genetic and functional studies on P. aeruginosa genome contains a disproportionately large number of genes predicted to encode outer membrane proteins involved in adhesion, motility, antibiotic efflux, virulence factor export, and environmental sensing by two-component systems which contributes host-specific pathogenesis [4]. Considering the genetic diversity of the $P$. aeruginosa genome, it is not surprising that it contains one of the highest percentages of predicted regulatory genes $(8,4 \%)$ of all bacterial genomes [5]. The understanding of transcription regulations in $P$. aeruginosa may offer some insight into how cohorts of virulence factors are coordinately expressed to influence pathogenesis in a range of pseudomonas infections [6]. An important molecular device to achieve sampling of environmental signals is the so-called two component regulatory system which allows to Pseudomoansaeruginosa contribution from initial of infection that starts with attachment to the host membrane and to growth in biofilm formation then spread to new appropriate fields to build up strong and disseminated infections. We focused on this mini review to two-component regulatory system contributes to rhamnolipid production under phosphate limited condition. Rhamnolipids are biodetergent and boosting the attachment to host membrane and growth in biofilm with the complex regulatory systems together to form a vigorous infection in Pseudomonas aeruginosa. The regulatory network for rhamnolipid production regulation on genetic level consists of very complex interaction of cell density dependent AHL-mediated QS and sigma factor $(\sigma)$ transcriptional regulation. Restriction in the availability of a number of nutrients, except the carbon source, is known to promote the production of RLs. For instance, under rich medium conditions the expression of RhlR is dependent on LasR, under phosphate-limiting conditions various transcriptional activators, including Vfr, RhlR, and the sigma factor $\sigma 54$, participatein the expression of RhlR from multiple promoters. P. aeruginosa during phosphate depletion; they included phosphate signaling (PhoB). The MvfR (multiple virulence factor regulator)-PQS pathway of quorum sensing, and pyoverdin iron acquisition system $[7,8]$. Rhamnolipid (rhl) is a secondary metabolite produced by P. aeruginosa, and it alters cell-cell and cell-surface interactions. It is a well-wetting agent for initial bacterial attachment as well as detachment Kim SK, et al [9]. Under P-limitation is lead to production of high volume of the rhl production and it helps to increase of addesive properties to the inert surfaces which are important in the hospital acquired infections especially for developing a medical device depending on P. aeruginosa 


\section{Open Access Journal of Mycology \& Mycological Sciences}

infections.

\section{Conflict of Interest}

A writer has no conflict of interest.

\section{Funding}

This publication has emerged by PhD thesis (Sebnem Bukavaz, 2015, The Effect of Rhamnolipid production on Pseudomonas aeruginosa Physiology, Ulm University, Ulm, Germany) funding by Marie Curie Actions.

\section{Acknowledgment}

Thanks to my Supervisor of the PhD thesis Dr. Frank Rosenau at Ulm University Institute of Pharmaceutical Biotechnology.

\section{References}

1. Rossi E, La Rosa R, Bartell JA, Marvig RL, Haagensen JAJ, et al. (2020) Pseudomonas aeruginosa adaptation and evolution in patients with cystic fibrosis. Nat Rev Microbiol.

2. Montero MM, López Montesinoz I, Knobel H, Molas E, Sorlí L, et al. (2020) Risk Factors for Mortality among Patients with Pseudomonas aeruginosa Bloodstream Infections: What Is the Influence of XDR Phenotype on Outcomes?. J Clin Med 9(2): 514.
3. Paterson DL, Isler B, Stewart A (2020) New treatment options for multiresistant gram negatives. Curr Opin Infect Dis 33(2): 214-223.

4. Dubern JF, Cigana C, De Simone M, Lazenby J, Juhas $M$, et al. (2015) Integrated whole-genome screening for Pseudomonas aeruginosa virulence genes using multiple disease models reveals that pathogenicity is host specific. Environ microbiol 17(11): 4379-4393.

5. Kung VL, Ozer EA, Hauser AR (2010) The accessory genome of Pseudomonas aeruginosa. Microbiol Mol Biol Rev 74(4): 621-641.

6. Turner KH, Vallet-Gely I, Dove SL (2009) Epigenetic control of virulence gene expression in Pseudomonas aeruginosa by a LysR-type transcription regulator. PLoS Genet 5(12): e1000779.

7. Blus-Kadosh I, Zilka A, Yerushalmi G, Banin E (2013) The effect of pstS and phoB on quorum sensing and swarming motility in Pseudomonas aeruginosa. PLoS One 8(9): e74444.

8. Guo Q, Kong W, Jin S, Chen L, Xu Y, et al. (2014) PqsRdependent and PqsR-independent regulation of motility and biofilm formation by PQS in Pseudomonas aeruginosa PA01. J Basic Microbiol 54(7): 633-643.

9. Kim SK, Lee JH (2016) Biofilm dispersion in Pseudomonas aeruginosa. J Microbiol 54(2): 71-85. 